\title{
Editorial on the European Society of Gastrointestinal Endoscopy (ESGE) and European Society of Gastrointestinal and Abdominal Radiology (ESGAR) guideline on clinical indications for CT colonography in the colorectal cancer diagnosis
}

\author{
Andrea Laghi $^{1} \cdot$ Emanuele Neri $^{2} \cdot$ Daniele Regge $^{3}$
}

Received: 20 January 2015 / Accepted: 31 March 2015 / Published online: 12 April 2015

(C) Italian Society of Medical Radiology 2015

\begin{abstract}
European Society of Gastrointestinal Endoscopy (ESGE)-European Society of Gastrointestinal and Abdominal Radiology (ESGAR) guideline was generated jointly by a team of researchers, including gastrointestinal radiologists and endoscopists, and represents the first full collaborative effort between the two specialties after years of turf battles involving CT colonography (CTC) and colonoscopy (CS). This guideline has a main educational purpose and it represents the attempt to find a consensus about the use of CTC in clinical practice based on the best current available evidence. Thus, it should not be considered as rules for establishing a legal standard of care. Main recommendations include the use of CTC as the radiological examination of choice for the diagnosis of colorectal neoplasia, the use of CTC in the case of incomplete CS, and the possible use of CTC as an acceptable and equally sensitive alternative for patients with symptoms suggestive of colorectal cancer (CRC), when CS is contraindicated or not possible. ESGE-ESGAR guideline does not recommend CTC for population screening, but considers that CTC may be proposed as a CRC screening test on an individual basis (opportunistic screening) provided the screenee is adequately informed about test characteristics, benefits and risks. With regard to patient management, referral for
\end{abstract}

Andrea Laghi

andrea.laghi@uniroma1.it

1 Department of Radiological Sciences, Oncology and Pathology, I.C.O.T. Hospital, Sapienza-Università di Roma, 04100 Latina, Italy

2 Diagnostic and Interventional Radiology, University of Pisa, Pisa, Italy

3 Candiolo Cancer Institute-FPO, IRCCS, Str. Prov. 142, km 3.95, Candiolo, 10060 Turin, Italy endoscopic polypectomy in patients with at least one polyp $\geq 6 \mathrm{~mm}$ in diameter detected at CTC is recommended, considering surveillance only in case polyp removal is not possible. Knowledge about CTC is in continuous evolution and this means that a revision might be necessary in the future as new data appear.

Keywords Computed tomography colonography · Virtual colonoscopy · CT colonography, indications . CT colonography, guideline

The twentieth anniversary of CT colonography (CTC) (1994-2014) sets another milestone in the history of this technique, following the publications of the first two randomized clinical trials on asymptomatic subjects [1] and symptomatic patients [2,3], respectively, and the updated edition of guidelines on CTC examination protocols [4]. In fact, the first guideline on indications for CTC was recently and simultaneously published on European Radiology [5], the official journal of the European Society of Radiology (ESR) and its sub-specialty societies, in this case the European Society of Gastrointestinal and Abdominal Radiology (ESGAR), and on Endoscopy [6], the official journal of the European Society of Gastrointestinal Endoscopy (ESGE). This guideline, the result of the collaboration between ESGAR and ESGE was generated jointly by a team of researchers, including gastrointestinal radiologists and endoscopists, and represents the first full collaborative effort between the two specialties after years of turf battles involving CTC and Colonoscopy (CS). Simultaneous publication in the respective leading European journals, and particularly, in the endoscopic journal, is an extremely important signal towards the full implementation of CTC in the gastroenterological community, also in centres, which are still reluctant. 
ESGE-ESGAR guideline gives an updated and shared view about CTC in different situations encountered during clinical practice and related to colorectal cancer diagnosis.

The first statement, on one side, recommends CTC as the radiological examination of choice for the diagnosis of colorectal neoplasia, and on the other side, does not recommend barium enema (BE) in this setting any more. Although obvious in academic and non-academic referral centres, the use of CTC is still far to be implemented in local environments where turf battles still exist, because radiologists are often not trained and therefore may not have adequate expertise to implement CTC in the local practice.

Reasons for discouraging the use of BE are plenty, but probably unknown to the medical community outside research centres. With the term medical, we refer not at radiologists and GI specialists, but particularly to general practitioners, who are in many cases unaware of the advantages of CTC in comparison with BE and the absolutely clear disadvantages of $\mathrm{BE}$, namely lower sensitivity and specificity than CTC for detecting colorectal polyps $>$ or $=6 \mathrm{~mm}$, which are the lesions recommend for polypectomy [7], higher patient discomfort [8] and higher level of radiation exposure [9].

The most important indication for CTC remains incomplete CS, to be performed preferably on the same day. This is technically feasible, even with the use of faecal tagging [10], unless reason for stopping CS was poor bowel preparation. In that case, the exam needs to be rescheduled on a different day with a new bowel preparation. Delay of CTC for approximately 2 weeks should also be considered after an endoscopic resection [5, 6]. If the reason for incomplete CS was an obstructing colorectal cancer, good clinical practice includes the use of intravenous contrast medium to perform a complete pre-operative staging of the neoplastic lesion [11]. Although incomplete CS might seem, to a non-expert observer, a minor indication for CTC, it is not. In fact, rate of incomplete CS is relatively high and it is in relation with the skill of the colonoscopist and the age of the population. In a recent Italian survey, caecal intubation rate was $80.7 \%$ [12]. Moreover, more and more frequently, elderly and frail patients, with significant comorbidities and/or in therapy with anticoagulant drugs, are immediately referred to CTC after a first unsuccessful attempt with CS. This is reasonable since in this sub-group of patients risks of either perforation [13] or bleeding [14] during CS are extremely high and diagnostic accuracy of CTC is absolutely adequate to either confirm or rule out CRC.

Thanks to the recent publication of the data of SIGGAR trial [2, 3], a randomized clinical trial comparing CTC diagnostic accuracy for CRC or significant $(\geq 1 \mathrm{~cm}$ ) polyps with either CS or BE in two different arms, ESGE/ESGAR recommends CTC as an acceptable and equally sensitive alternative for patients with symptoms suggestive of CRC, when CS is contraindicated or not possible. This opens the doors of CTC to symptomatic patients, considering the extremely low positive predictive value of alarm symptoms [15]. Nonetheless, the ability to detect and remove early colonic lesions, the possibility to perform histologic analysis of diagnosed CRC and the higher sensitivity for the detection of colitis and anal pathology remain potential advantages of CS over CTC. On the other side, diverticulosis, one of the major causes of nonspecific abdominal symptoms in the elderly is better diagnosed with CTC [2]. Further research is needed in this area to better stratify the patients to refer those with higher probability to find a lesion to CS and the others to CTC.

Another important problem encountered in clinical practice is the need for endoscopic polypectomy after a lesion is detected at CTC. ESGE-ESGAR recommends the referral to polypectomy in case of polyps $\geq 6 \mathrm{~mm}$ in diameter detected at CTC, and suggests CTC surveillance only in those who do not undergo polypectomy for any reason. This statement is based on consistent and robust data about the high rate of advanced adenomas (AAs) among large $(\geq 10 \mathrm{~mm}$ ) polyps [16] and the still unknown prevalence of AAs among small (6-9 $\mathrm{mm}$ ) lesions [17].

The position of the two societies about the use of CTC for CRC screening is clear and it is in line with European Union (EU) recommendations, suggesting faecal occult blood test as the method of choice for CRC screening in Europe [18]. For this reason, ESGE and ESGAR do not recommend CTC as a primary test for population screening, but since there is clear evidence of the high diagnostic accuracy of CTC, both societies consider that CTC may be proposed as a CRC screening test on an individual basis provided the screenee is adequately informed about test characteristics, benefits, and risks. This statement follows the recommendation of the American Cancer Society in 2008 that included CTC in the list of optional screening tests for asymptomatic average-risk individuals [19].

Concerning screening for individuals with a positive firstdegree family history of CRC, the evidence is still limited [20] and at the moment clear recommendations cannot be provided. This is similar to the potential use of CTC for surveillance after curative-intent resection of CRC or in patients with high-risk polyps in surveillance after polypectomy. In both situations, CS is still the examination of choice and CTC should be considered only if CS is unfeasible.

Finally, CTC is contraindicated in patients with active colonic inflammation and in those who have recently undergone colorectal surgery.

Other statements of great interest for those performing or willing to perform CTC are available in the full papers, downloadable from the web.

ESGE-ESGAR guideline represents the first attempt of a radiological and a gastroenterological endoscopic society 
to find a consensus about the use of CTC in clinical practice based on the best current available evidence. This guideline has a main educational purpose and it should not be considered as rules for establishing a legal standard of care. Knowledge about CTC is in continuous evolution and this means that a revision might be necessary in the future as new data appear. In any case, we wish that this publication will help overcome turf battles between CTC and CS, still present in local practice.

Conflict of interest The authors declare that they have no conflict of interest.

Ethical standards This article does not contain any studies with human participants or animals performed by any of the authors.

\section{References}

1. Stoop EM, de Haan MC, de Wijkerslooth TR, Bossuyt PM, van Ballegooijen M, Nio CY, van de Vijver MJ, Biermann K, Thomeer M, van Leerdam ME, Fockens P, Stoker J, Kuipers EJ, Dekker E (2012) Participation and yield of colonoscopy versus non-cathartic CT colonography in population-based screening for colorectal cancer: a randomised controlled trial. Lancet Oncol 13:55-64

2. Atkin W, Dadswell E, Wooldrage K, Kralj-Hans I, von Wagner C, Edwards R, Yao G, Kay C, Burling D, Faiz O, Teare J, Lilford RJ, Morton D, Wardle J, Halligan S, SIGGAR Investigators (2013) Computed tomographic colonography versus colonoscopy for investigation of patients with symptoms suggestive of colorectal cancer (SIGGAR): a multicentre randomised trial. Lancet 381:1194-1202

3. Halligan S, Wooldrage K, Dadswell E, Kralj-Hans I, von Wagner C, Edwards R, Yao G, Kay C, Burling D, Faiz O, Teare J, Lilford RJ, Morton D, Wardle J, Atkin W, SIGGAR Investigators (2013) Computed tomographic colonography versus barium enema for diagnosis of colorectal cancer or large polyps in symptomatic patients (SIGGAR): a multicentre randomised trial. Lancet 381:1185-1193

4. Neri E, Halligan S, Hellström M, Lefere P, Mang T, Regge D, Stoker J, Taylor S, Laghi A, ESGAR CT colonography Working Group (2013) The second ESGAR consensus statement on CT colonography. Eur Radiol 23:720-729

5. Spada C, Stoker J, Alarcon O, Bellini D, Bretthauer M, De Haan MC, Dumonceau JM, Ferlitsch M, Halligan S, Helbren E, Hellstrom M, Kuipers EJ, Lefere P, Mang T, Neri E, Petruzziello L, Plumb A, Regge D, Taylor SA, Hassan C, Laghi A (2015) Clinical indications for CT colonography: European Society of Gastrointestinal Endoscopy (ESGE) and European Society of Gastrointestinal and Abdominal Radiology (ESGAR) guidelines. Eur Radiol 25:331-345

6. Spada C, Stoker J, Alarcon O, Bellini D, Bretthauer M, De Haan MC, Dumonceau JM, Ferlitsch M, Halligan S, Helbren E, Hellstrom M, Kuipers EJ, Lefere P, Mang T, Neri E, Petruzziello L, Plumb A, Regge D, Taylor SA, Hassan C, Laghi A (2014) Clinical indications for CT colonography: European Society of Gastrointestinal Endoscopy (ESGE) and European Society of Gastrointestinal and Abdominal Radiology (ESGAR) guidelines. Endoscopy 46:897-915

7. Sosna J, Sella T, Sy O, Lavin PT, Eliahou R, Fraifeld S, Libson E (2008) Critical analysis of the performance of double-contrast barium enema for detecting colorectal polyps $>$ or $=6 \mathrm{~mm}$ in the era of CT colonography. AJR Am J Roentgenol 190:374-385

8. von Wagner C, Smith S, Halligan S, Ghanouni A, Power E, Lilford RJ, Morton D, Dadswell E, Atkin W, Wardle J, Investigators SIGGAR (2011) Patient acceptability of CT colonography compared with double contrast barium enema: results from a multicentre randomised controlled trial of symptomatic patients. Eur Radiol 21:2046-2055

9. Neri E, Faggioni L, Cerri F, Turini F, Angeli S, Cini L, Perrone F, Paolicchi F, Bartolozzi C (2010) CT colonography versus double-contrast barium enema for screening of colorectal cancer: comparison of radiation burden. Abdom Imag 35:596-601

10. Chang KJ, Rekhi SS Jr, Anderson SW, Soto JA (2011) Fluid tagging for CT colonography: effectiveness of a 2-hour iodinated oral preparation after incomplete optical colonoscopy. J Comput Assist Tomogr 35:91-95

11. Park SH, Lee JH, Lee SS, Kim JC, Yu CS, Kim HC, Ye BD, Kim MJ, Kim AY, Ha HK (2012) CTcolonography for detection and characterisation of synchronous proximal colonic lesions in patients with stenosing colorectal cancer. Gut 61:1716-1722

12. Radaelli F, Meucci G, Minoli G, Italian Association of Hospital Gastroenterologists (AIGO) (2008) Colonoscopy practice in Italy: a prospective survey on behalf of the Italian Association of Hospital Gastroenterologists. Dig Liver Dis 40:897-904

13. Gatto NM, Frucht H, Sundararajan V, Jacobson JS, Grann VR, Neugut AI (2003) Risk of perforation after colonoscopy and sigmoidoscopy: a population-based study. J Natl Cancer Inst 95:230-236

14. Ko CW, Riffle S, Michaels L, Morris C, Holub J, Shapiro JA, Ciol MA, Kimmey MB, Seeff LC, Lieberman D (2010) Serious complications within 30 days of screening and surveillance colonoscopy are uncommon. Clin Gastroenterol Hepatol 2:166-173

15. Jellema $P$, van der Windt DA, Bruinvels DJ, Mallen CD, van Weyenberg SJ, Mulder CJ, de Vet HC (2010) Value of symptoms and additional diagnostic tests for colorectal cancer in primary care: systematic review and meta-analysis. BMJ 340:c1269

16. Hassan C, Pickhardt PJ, Kim DH, Di Giulio E, Zullo A, Laghi A, Repici A, Iafrate F, Osborn J, Annibale B (2010) Systematic review: distribution of advanced neoplasia according to polyp size at screening colonoscopy. Aliment Pharmacol Ther 31:210-217

17. Kolligs FT, Crispin A, Graser A, Munte A, Mansmann U, Göke B (2013) Risk factors for advanced neoplasia within subcentimetric polyps: implications for diagnostic imaging. Gut 62:863-870

18. von Karsa L, Patnick J, Segnan N (2012) European guidelines for quality assurance in colorectal cancer screening and diagnosis-executive summary. Endoscopy 44(3):SE1-SE8

19. Levin B, Lieberman DA, McFarland B, Smith RA, Brooks D, Andrews KS, Dash C, Giardiello FM, Glick S, Levin TR, Pickhardt P, Rex DK, Thorson A, Winawer SJ; American Cancer Society Colorectal Cancer Advisory Group, US Multi-Society Task Force, American College of Radiology Colon Cancer Committee (2008) Screening and surveillance for the early detection of colorectal cancer and adenomatous polyps, 2008: a joint guideline from the American Cancer Society, the US Multi-Society Task Force on Colorectal Cancer, and the American College of Radiology. CA Cancer J Clin 58:130-160

20. Regge D, Laudi C, Galatola G, Della Monica P, Bonelli L, Angelelli G, Asnaghi R, Barbaro B, Bartolozzi C, Bielen D, Boni L, Borghi C, Bruzzi P, Cassinis MC, Galia M, Gallo TM, Grasso A, Hassan C, Laghi A, Martina MC, Neri E, Senore C, Simonetti G, Venturini S, Gandini G (2009) Diagnostic accuracy of computed tomographic colonography for the detection of advanced neoplasia in individuals at increased risk of colorectal cancer. JAMA 301:2453-2461 